\title{
La démarche du Population Council: Promouvoir la santé, la sécurité et la productivité transitions vers l'âge adulte
}

Population Council

Follow this and additional works at: https://knowledgecommons.popcouncil.org/departments_sbsr-pgy

Part of the Demography, Population, and Ecology Commons, Family, Life Course, and Society Commons, Gender and Sexuality Commons, International Public Health Commons, and the Medicine and Health Commons How does access to this work benefit you? Let us know!

\section{Recommended Citation}

"La démarche du Population Council: Promouvoir la santé, la sécurité et la productivité transitions vers l'âge adulte," Promouvoir la santé, la sécurité et la productivité transitions vers l'âge adulte. New York: Population Council, 2011. 


\section{L'enjeu}

\section{L'adolescence est une} phase essentielle du développement humain au cours de laquelle le décor de la vie adulte est planté.

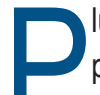

lus d'1,5 milliards de personnes âgées de 10 à 24 ans dans les pays en développement passent de l'enfance aux rôles et responsabilités de la vie d'adulte - devenant employés, citoyens, époux et parents - au cours d'une période de changement global sans précédent. Les jeunes générations à suivre seront d'autant plus nombreuses. Sans investissements appropriés consacrés à leur santé et leur éducation ainsi qu'à leurs possibilités de générer des moyens de subsistance productifs, les perspectives d'avenir de la jeune génération seront limitées. En Afrique, où la moitié des nouvelles infections par le VIH touchent les personnes âgées de 15 à 24 ans, en majorité des jeunes femmes, la jeune génération se heurte à des difficultés particulières. L'avenir d'un grand nombre de nations, ainsi que la réalisation de la plupart des Objectifs du Millénaire pour le développement fixés par les Nations unies, dépendent de la manière dont ces adolescents parviendront à se frayer un chemin dans cette phase de leur cycle de vie.

\section{La démarche du Population Council}

\section{L'intérêt particulier porté aux adolescents par le Population Council date des années 1990 et s'ac- compagne d'un engage- ment à ouvrir le champ des études et des programmes consacrés aux adolescents en élargissant le simple domaine de la sexualité, de}

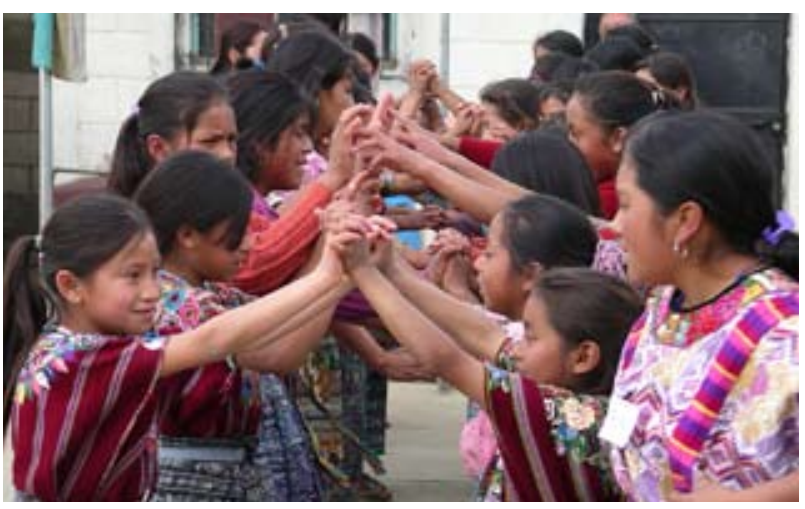

Le programme Abriendo Oportunidades crée des espaces sécurisés et met des tutrices à la disposition des jeunes filles Maya.

la santé génésique et des comportements aux grandes questions sociales et économiques qui sous-tendent la santé et le bien-être des adolescents.

En 1998, avec la publication de The Uncharted Passage - une analyse comparative de la situation des adolescentes dans les pays en développement - le programme de travail actuel fut lancé. La démarche du Council se caractérise par quatre particularités :

\footnotetext{
Une attention constante prêtée aux implications des sexospécificités au cours de l'adolescence, notamment les besoins des filles dans les pays les moins développés. Dans ce type de milieu, les possibilités offertes aux jeunes filles sont souvent limitées elles sont fréquemment victimes de violences sexistes, sont exposées au risque du mariage d'enfants et sont touchées par l'épidémie de $\mathrm{VIH}$ de manière disproportionnée.

Une approche multisectorielle qui implique les différentes facettes sociétales agissant sur la vie des adolescentes: les cercles familiaux et les responsables locaux ; les
}

garçons et les hommes ; les organisations non gouvernementales et locales; les secteurs de la santé et de l'éducation. Les mauvais états de santé à l'adolescence ne découlent pas seulement de facteurs physiologiques mais sont également le résultat de l'isolement social et de la vulnérabilité économique des jeunes gens — en particulier des jeunes filles.

- Une préoccupation particulière concernant l'inégalité - reposant sur le sexe, la catégorie socioéconomique, la résidence en zone urbaine/rurale et l'accès à l'économie moderne - qui s'intensifie pendant l'adolescence.

- Un engagement à mener des recherches politiques documentées en parallèle à un programme de développement et d'évaluation, en veillant à ce que l'analyse réglementaire soit rapidement appliquée aux programmes et politiques sur le terrain. 


\section{Théorie du changement}

La théorie du changement définie par le Council confirme que les fondements de la vie productive - qui peuvent impliquer un mariage, une première naissance, un emploi, la citoyenneté et la suffisance économique - se forment à l'adolescence. Les composantes de ces fondements comprennent les aptitudes personnelles et sociales, l'éducation financière, la santé sexuelle et génésique, les compétences de négociation et l'alphabétisation. Cette période est également celle durant laquelle les conditions de pauvreté peuvent être accrues ou surmontées, et les premiers actes de violence envers les femmes perpétrés. Ainsi, notre intérêt est principalement porté sur le développement des ressources sociales et économiques des adolescents, qui comptent parmi les populations les plus vulnérables, afin de garantir des transitions sûres et productives vers ces rôles d'adulte. Ces ressources sont acquises et ne peuvent être confisquées aux jeunes gens ; si les avantages de ces ressources ne sont pas toujours compris avant l'âge adulte (souvent plusieurs années plus tard), des résultats intermédiaires peuvent néanmoins être appréciés.

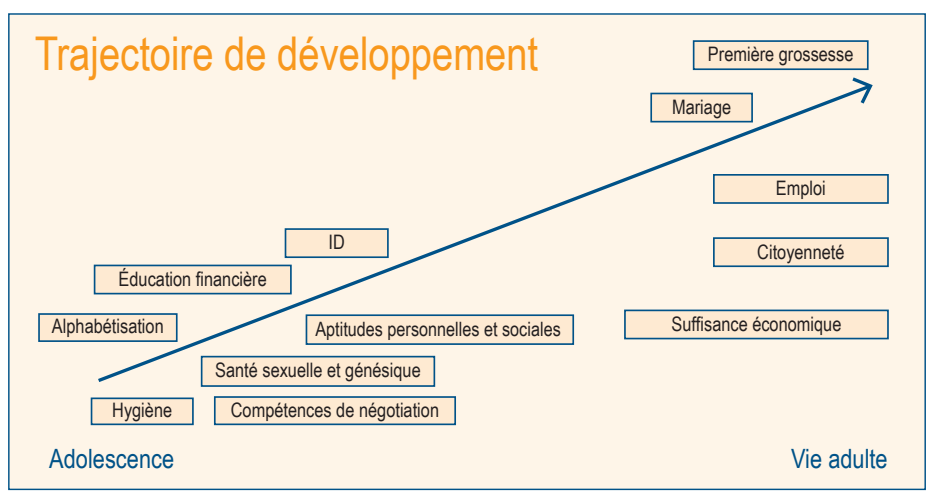

\section{Stratégie}

Le Council utilise des données afin de mieux comprendre la diversité des vies et des expériences adolescentes et d'identifier les adolescents les plus vulnérables et les plus défavorisés. II travaille ensuite en coopération avec des partenaires locaux pour élaborer des programmes de développement des ressources de ces adolescents, évalue les changements qui surviennent au niveau individuel et collectif, et favorise l'élargissement des stratégies efficaces. Tout au long de ce parcours, des outils sont élaborés pour mener des recherches et proposer des programmes applicables sur le terrain. L'engagement auprès des responsables politiques - aux niveaux local, national et international imprègne ce travail à chaque étape afin de garantir le développement de politiques destinées à répondre aux besoins et aux expériences de cette cohorte essentielle de la population.

Recueillir, analyser et diffuser les données concernant la diversité des vies et des expériences adolescentes

Le Council a pour priorité d'exploiter les données existantes et de recueillir de nouvelles données afin de mettre en lumière les influences caractéristiques de l'âge, du sexe, de la scolarité, de la situation familiale, du mode de vie et du lieu de résidence en zone urbaine/rurale sur l'expérience adolescente.

L'analyse secondaire des listes de foyers recueillies lors des Enquêtes démographiques et de santé fournit des informations élémentaires concernant les adolescents qui résident dans ces foyers. Ces informations sont résumées dans 55 guides-pays créés par le Population Council : The Adolescent Experience In-depth: Using Data to Identify and Reach the Most Vulnerable Young People [L'expérience adolescente en détails : utilisation des données pour identifier et entrer en contact avec les jeunes populations les plus vulnérables]. Cette série a pour objet de fournir aux décideurs de tous horizons - issus de gouvernements, d'organisations non gouvernementales (ONG) et de groupes de pression - des données relatives à la situation des adolescents et des jeunes femmes. La tranche d'âge concernée est celle de 10 à 24 ans. Ces données sont présentées sous forme de graphiques, de tableaux et de cartes (dans la mesure du possible) afin de fournir plusieurs formats et ainsi rendre les informations accessibles à un public diversifié (TA $\left.n^{\circ} 10\right)$.

Enquêtes représentatives nationales et infranationales. Les données existantes fournissent rarement les informations détaillées obtenues en interrogeant directement les adolescents. Le Council a mis en œurre des enquêtes partout dans le monde afin de se faire une idée plus précise de la vie des adolescents. Des enquêtes nationales ont été menées au Bangladesh, en Égypte, en Éthiopie, en Inde et au Pakistan, tandis que des études longitudinales réalisées au Malawi et en Afrique du Sud fournissent la possibilité unique de suivre les adolescents dans le temps.

Présenter les données de manière conviviale. Une grande quantité de données recueillies dans le monde ne sont jamais analysées. Même les données analysées sont souvent présentées de telle sorte que seuls des statisticiens professionnels peuvent les interpréter. Le Council s'efforce de rendre ces données accessibles à divers types de public à des fins de planification, de mise en œuvre et d'évaluation des programmes ainsi que d'analyse politique. Outre la création de cartes à l'aide de statistiques, nous utilisons de plus en plus la cartographie comme exercice participatif permettant aux adolescents des communautés locales de cartographier et de décrire le contexte de leur vie, pour définir les problèmes, proposer des mécanismes de lutte et concevoir des stratégies de développement ( $\left.\operatorname{TAn}^{\circ} 30\right)$.

\section{Créer des outils innovants pour le terrain}

Dans le cadre de son analyse de données et de la mise en œuvre de son programme, le Council crée des ressources et des outils innovants destinés à l'usage d'autrui. L'un des outils importants de recueil d'informations auprès des adolescents concernant les sujets sensibles a été l'auto-entretien audio assisté par ordinateur (ACASI). Le Council a développé son propre logiciel d'utilisation de cet outil et a ainsi amélioré encore davantage la capacité de normalisation, la confidentialité, la flexibilité linguistique et la sécurité du recueil de données.

Notre travail à grande échelle de conception de programmes et d'intervention a permis de produire deux ressources essentielles :

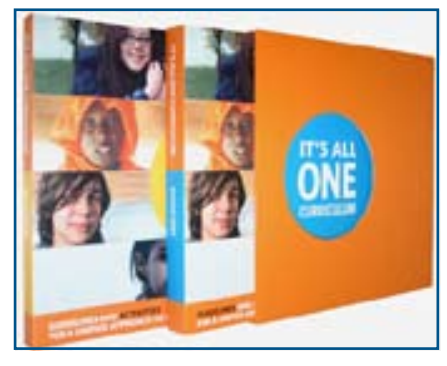

It's All One Curriculum, un programme sur deux colonnes qui place les questions de sexospécificité et les droits de l'homme au cœur de l'éducation sexuelle et de l'information sur le VIH, avec en parallèle 54 activités qui engagent les jeunes et favorisent le développement des compétences de réflexion critique. Disponible en anglais, en espagnol et en français. www.itsallone.org 


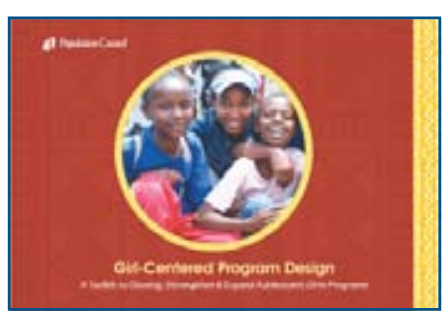

Girl-Centered Program Design: A

Toolkit to Develop, Strengthen, and Expand Adolescent Girls Programs. [Conception d'un programme centré sur les filles : boîte à outils pour l'élaboration, le renforcement et l'expansion de programmes destinés aux adolescentes.] Cette boîte à outils concerne principalement la structure, le contenu et l'évaluation des programmes destinés aux jeunes filles. Cet outil pratique permet d'amorcer ou de renforcer les programmes destinés aux jeunes filles et est disponible en anglais, en espagnol et en français. www.popcouncil.org/publications/books/2010_AdolGirlsToolkit.asp

Utiliser les espaces sécurisés pour développer les ressources en vue de construire une vie d'adulte saine et productive

La portée et la couverture limitées des programmes jeunesse existants ainsi que la priorité donnée aux informations et aux services sanitaires n'ont pas permis de répondre suffisamment aux besoins de nombreux adolescents. Ces jeunes gens sont parfois déscolarisés, loin de chez eux, mariés ou piégés dans un cycle d'exploitation par le travail et de pauvreté. Le Population Council a centré ses premiers efforts sur le développement de ressources destinées aux adolescentes qui sont exposées à des risques disproportionnés. Des interventions expérimentales ont été élaborées en collaboration avec les partenaires locaux pour fournir aux adolescentes des lieux de réunion protégés. Dans ces "espaces sécurisés», les jeunes filles nouent des relations saines avec leurs paires et leurs tutrices, gagnent en assurance, acquièrent des connaissances et assimilent de nouvelles compétences.

\section{Enseignements tirés}

\section{Quelle cible parvenons-nous à atteindre ?}

Les interventions du Council ont démontré qu'il est possible d'entrer en contact avec des adolescents vulnérables qui n'ont jamais bénéficié des investissements opérés en faveur de la jeunesse. Par le biais de stratégies de recrutement ciblées, ces interventions ont identifié et recruté les populations visées.

- Les jeunes filles exposées au risque de mariage précoce sont souvent maintenues à l'écart des manifestations publiques mais ont été recrutées lors d'interventions culturellement acceptables au Bangladesh, au Burkina Faso, en Égypte, en Éthiopie, au Guatemala, en Inde et au Kenya, qui ont permis de repousser considérablement l'âge auquel elles se marient (TA $n^{\circ} 13 ; 9 ; 12 ; 20 ; 5 ; 2 ; 19 ; 29$ ).

Les jeunes filles mariées comptent parmi les adolescentes les plus difficiles à atteindre. Une fois mariée, la jeune fille s'installe souvent dans le foyer familial de son époux, où ses relations sociales sont très limitées et où elle vit avec un homme souvent bien plus âgé qu'elle. Des projets mis en place au Burkina Faso, en Éthiopie et en Inde ont permis de sortir ces jeunes filles de l'ombre, de créer des liens entre elles pour développer un soutien social mutuel et de leur fournir un accès aux services $\left(T A n^{\circ} 9 ; 20 ; 8\right)$. Le projet First Time Parents en Inde engageait la participation des deux partenaires et était centré sur les aspects positifs de la maternité en réévaluant les rôles et les attentes de chaque sexe pour les soins des enfants, la cuisine, la collecte d'eau, etc.

- Les employées domestiques des foyers urbains travaillent dans des conditions extrêmement pénibles, touchent un salaire de misère ou inexistant, effectuent des horaires éreintants et sont fréquemment victimes d'abus sexuels. En Éthiopie, où de nombreuses jeunes filles se réfugient à Addis-Abeba après s'être enfuies des zones rurales pour échapper au mariage, les employées domestiques ont été identifiées au moyen d'une stratégie de recrutement en porte-à-porte. Les négociations avec les employeurs ont donné lieu à la création d'un programme d'espaces sécurisés, le programme Biruh Tesfa $\left(\mathrm{TA} \mathrm{n}^{\circ} 21\right)$. Ainsi, dans un bâtiment public des quartiers défavorisés d'Addis-Abeba, des jeunes filles âgées de 10 à 19 ans rencontrent chaque semaine leurs tutrices pour acquérir les ressources sociales et économiques qui leur permettront de reconstruire leur vie.

- Jeunes adolescentes. La première vague d'interventions visait les jeunes filles âgées de 15 ans et plus. Mais il s'est rapidement révélé nécessaire d'intervenir bien avant la période où les jeunes filles sont confrontées à certains événements (mariage d'enfants, déscolarisation, premier rapport sexuel, etc.). Ces projets touchent de plus en plus de jeunes filles âgées de 10 à 14 ans.

\section{Jeunes filles exposées au risque d'infection par le VIH. Les} adolescentes restent les personnes les plus touchées par les infections par le VIH. Les programmes d'espaces sécurisés développent les relations positives entre pairs, les compétences de négociation et les ressources économiques qui permettront aux jeunes filles d'éviter tout comportement indésirable et/ou hasardeux.

\section{Que fournissons-nous?}

Outre les ressources sociales créées dans les espaces sécurisés, de nombreuses interventions sont centrées sur le développement des possibilités financières, des plans d'épargne, des opportunités de création de moyens de subsistance et de l'information sur la santé sexuelle et génésique :

- Le programme Siyakha Nentsha en Afrique du Sud tire parti des taux élevés de scolarisation dans la région du KwaZulu Natal et intervient auprès de 1100 filles et garçons dans les écoles. L'une des composantes de ce programme est l'éducation financière et le contenu du programme a été certifié par le gouvernement de sorte que la validation de ce programme assure à ces jeunes gens une référence marchande (TA $\left.n^{\circ} 4\right)$.

- Par l'intermédiaire de clubs d'adolescents dans les zones rurales et les écoles techniques non officielles en zones urbaines, le programme Kishori Abhijan au Bangladesh est destiné aux jeunes filles âgées de 15 à 19 ans afin de promouvoir l'éducation financière par l'entremise de relations de tutorat. Nous évaluons actuellement la valeur de l'intégration d'un système de tutorat dans les démarches d'éducation financière traditionnelles $\left(\operatorname{TA~}^{\circ}{ }^{13}\right)$.

- Le projet «Safe and Smart Savings» est une extension de l'un des tous premiers programmes d'espaces sécurisés mis en place au Kenya : Binti Pamoja. Localisé dans le bidonville de Kibera, ce programme s'étend audelà de l'éducation financière pour se muer en un véritable programme bancaire mis en place en partenariat avec deux banques de Nairobi. $\mathrm{Ce}$ programme est actuellement en cours de développement en Ouganda. Avec 2300 jeunes actuellement concernés, la phase complète de lancement devrait permettre d'en atteindre près de 20000 (TA n 29 ).

\section{Comment ces programmes sont-ils reliés aux autres services ?}

Ces interventions ne fournissent pas directement des services sanitaires, mais mettent en relation les participants avec les services existants au sein de la communauté au moyen de visites d'exposition, de collaborations officielles et de campagnes de sensibilisation. 
- Moyens de subsistance/épargne. Au Kenya, ce programme fonctionne en partenariat avec des banques commerciales pour garantir aux jeunes filles un accès aux services financiers du secteur privé (TA $\left.n^{\circ} 29\right)$. En Afrique du Sud, le programme sensibilise les jeunes aux programmes publics d'assistance financière pour lesquels un grand nombre d'adolescents remplissent les conditions d'admissibilité mais savent rarement comment y accéder.

- Éducation. Le programme Ishraq en Égypte est un programme de «deuxième chance» qui a pour objectif d'entrer en contact avec les jeunes filles déscolarisées et d'assurer leur intégration dans des écoles publiques à l'issue du programme. Une fois ce projet pilote achevé, $92 \%$ des jeunes filles qui s'étaient présentées à l'examen l'ont réussi et se sont engagées dans un cursus scolaire officiel ( $\left.T A{ }^{\circ} 12\right)$.

- Services de lutte contre les violences basées sur le genre. Si ces programmes n'ont pas été élaborés dès le début comme des programmes de lutte contre les violences basées sur le genre, les tutrices intervenant dans les programmes d'espaces sécurisés ont noté que la question des violences émergeait de façon manifeste sur tous les sites d'interventions. Les espaces réservés aux filles sont devenus des espaces protégés dans lesquels elles peuvent parler de ce sujet, apprendre à connaître leurs droits et mettre en commun des stratégies de lutte. Ces programmes évoluent constamment en fonction des lieux de prévention de la violence.

\section{Ces programmes peuvent-ils être élargis ?}

Le développement d'un programme dépend majoritairement de l'existence d'un gouvernement, d'une ONG ou d'organisations religieuses ou communautaires disposant des capacités nécessaires pour mettre en œuvre le programme auprès d'une population nombreuse et diversifiée. Les modèles d'élargissement sont considérablement différents selon le contexte, mais les caractéristiques communes à tous sont les suivantes : 1) engagement rapide auprès d'éventuels partenaires de développement et 2) attention portée aux coûts et à la rationalisation du programme.

- Le projet Biruh Tesfa mené dans les zones insalubres d'Éthiopie a fondé un partenariat avec le gouvernement dès le lancement du programme. Installé dans des salles mises à disposition par les collectivités locales qebelé, le programme touche désormais 16500 jeunes filles déscolarisées et est en cours de développement dans 12 villes supplémentaires en vue de profiter à quelques 30000 jeunes filles supplémentaires. Les salaires des tutrices ont également été alignés sur le niveau de rémunération d'un travailleur social public, ce qui laisse la possibilité d'une évolution en puisant dans les ressources publiques $\left(\operatorname{TA~}^{\circ}{ }^{\circ} 21\right)$.

- Dans les zones rurales de la Haute-Égypte, le programme Ishraq intervient auprès de 1800 jeunes filles âgées de 12 à 15 ans pour favoriser l'assiduité à l'école et accroître le niveau d'éducation sanitaire. Ce programme bénéficie aujourd'hui du soutien solide de la communauté et du gouvernement. II se trouve désormais dans sa phase d'élargissement et devrait bientôt compter parmi les programmes parrainés par le gouvernement $\left(T A n^{\circ} 12\right)$.

- Le programme Abriendo Oportunidades au Guatemala concerne plus de 1260 jeunes filles Maya des zones rurales âgées de 8 à 18 ans. Ces programmes sont appliqués dans des salles et des écoles locales et utilisent un modèle de «tutorat en cascade» afin de permettre aux jeunes filles d'évoluer vers des responsabilités de plus en plus importantes dans le cadre du programme. Les autorités locales, ainsi que les ONG et le secteur commercial privé, sont de plus en plus engagés et fournissent les attaches nécessaires au développement de la viabilité et de l'institutionnalisation. Outre l'élargissement du programme en lui-même, on observe actuellement une adhésion et une reproduction du modèle Abriendo par le public, les ONG et les partenaires du secteur privé au Guatemala. Ce programme est sur le point de devenir le premier programme national destiné aux jeunes filles autochtones de cette région ( $\left.\operatorname{TA~}^{\circ}{ }^{\circ} 5\right)$.

- Le programme Siyakha Nentsha en Afrique du Sud est déjà un cursus agréé et le Ministère de l'éducation est désireux d'étendre le programme à la région de KwaZulu Natal et au-delà (TA $\left.n^{\circ} 4\right)$.

- Le programme Berhane Hewan mis en place dans les régions rurales d'Éthiopie touche 12000 jeunes filles mariées et non mariées, âgées de 10 à 19 ans, dans une région où la moitié des jeunes filles sont mariées dès l'âge de 15 ans. Les structures qebelé existantes dans les villages font office de lieux de réunion et, grâce au soutien du gouvernement, le programme devrait bientôt concerner quelques 72000 jeunes filles ( $T A n^{\circ} 20$ ).

- Le programme Kishori Abhijan vise à réduire les taux d'abandon scolaire, à accroître l'activité économique indépendante des jeunes filles et élever l'âge auquel les jeunes filles se marient. Une initiative conjointe menée par le Population Council, l'UNICEF, I'UNFPA, le Bangladesh Institute of Development Studies (BIDS), le Bangladesh Rural Advancement Committee (BRAC) et le Centre for Mass Education in Science (CMES), ce programme allie une formation centrée sur les aptitudes personnelles et sociales et les moyens de subsistance avec le BRAC et le CMES, des ONG importantes et renommées qui ont mis en œuvre ce programme dans 14 circonscriptions rurales et ont recruté 15000 jeunes filles âgées de 13 à 22 ans. Au regard des résultats d'un projet pilote efficace évalué par le Population Council et le BIDS, la composante des aptitudes personnelles et sociales est en cours de développement grâce au travail déployé par le BRAC et le CMES en vue d'inscrire plus de 250000 jeunes filles dans 58 circonscriptions ( $\left.T A n^{\circ} 13\right)$.

\section{Quelle est la prochaine étape?}

La prochaine étape de ce travail consistera à démontrer de manière concrète l'impact des investissements opérés dans le développement des aptitudes personnelles et sociales et des ressources au cours de l'adolescence sur les résultats ultérieurs. Nous nous proposons de le faire en suivant les participantes dans le temps sur au moins deux projets en cours et en intégrant une approche longitudinale aux nouveaux projets. Après constatation de la nécessité d'intégrer le développement des ressources individuelles au contexte d'un environnement favorable, ces nouveaux projets s'intéresseront de plus près aux garçons et aux hommes, en donnant la priorité à une approche communautaire de prévention de la violence envers les femmes.

\section{Population Council}

Le Population Council fait évoluer les perspectives sur les questions essentielles de santé et de développement. Nous cherchons à comprendre les causes et les conséquences de l'inégalité entre les sexes et des disparités sociales qui s'installent au cours de l'adolescence. Nous fournissons des données en vue d'améliorer les programmes et les politiques mis en place sur le terrain qui permettent d'assurer des transitions efficaces et productives vers la vie adulte dans les pays en développement. www.popcouncil.org

() 2011 The Population Council, Inc.

4 - Rendez-vous sur www.popcouncil.org/publications/serialsbriefs/TABriefs.asp pour consulter tous les bulletins d'information de la série Promoting healthy, safe, and productive transitions to adulthood [Promouvoir des transitions saines, sécurisées et productives vers l'âge adulte]. 